\title{
Trivium
}

Revue franco-allemande de sciences humaines et sociales - Deutsch-französische Zeitschrift für Geistesund Sozialwissenschaften

32 | 2021

Institutions

\section{Les institutions et les exigences paradoxales de la modernité}

\author{
Jean-François Kervégan, Christian Schmidt et Benno Zabel
}

Traducteur : Pierre Rusch

\section{CpenEdition}

Journals

Édition électronique

URL : http://journals.openedition.org/trivium/7496

DOI : 10.4000/trivium.7496

ISBN : 1963-1820

ISSN : 1963-1820

\section{Éditeur}

Les éditions de la Maison des sciences de l'Homme

\section{Référence électronique}

Jean-François Kervégan, Christian Schmidt et Benno Zabel, « Les institutions et les exigences paradoxales de la modernité », Trivium [En ligne], 32 | 2021, mis en ligne le 28 janvier 2021, consulté le 18 mars 2021. URL : http://journals.openedition.org/trivium/7496 ; DOI : https://doi.org/10.4000/ trivium.7496

Ce document a été généré automatiquement le 18 mars 2021.

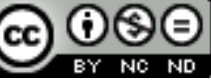

Les contenus des la revue Trivium sont mis à disposition selon les termes de la Licence Creative Commons Attribution - Pas d'Utilisation Commerciale - Pas de Modification 4.0 International. 


\title{
Les institutions et les exigences paradoxales de la modernité
}

\author{
Jean-François Kervégan, Christian Schmidt et Benno Zabel \\ Traduction : Pierre Rusch
}

1 Les institutions sont la forme que prend le pouvoir dans les sociétés différenciées modernes. Même si les sciences sociales d'aujourd'hui ne sont pas très à l'aise avec ce concept omniprésent, trop vague et trop polysémique à leur goût, elles sont pourtant unanimes à considérer que les institutions - que ce soit par l'attribution de rôles, par la structuration organisationnelle ou très banalement par des menaces de sanction dirigent l'agir et le jugement humain dans des voies prévisibles. Or cela s'appelle «le pouvoir ", à savoir une capacité d'amener des individus à interagir et à coopérer d'une manière prédéterminée. Les sociétés modernes se distinguent par le fait que le pouvoir implique de moins en moins l'aptitude à disposer immédiatement des individus ou des groupes sociaux. Désormais, les vecteurs de pouvoir sont souvent des structures dont l'existence peut être décrite comme un fait social. Car de telles structures (qui peuvent être articulées sur un mode hiérarchique ou sur un mode hétérarchique) résultent certes elles-mêmes de décisions et d'actions humaines coordonnées, et ne sont maintenues en vie que par de telles actions. Mais une fois venues à l'existence - « au monde »-, elles précèdent les intentions des individus, elles influencent, forment et déterminent celles-ci, tout comme une langue précède la parole individuelle, même si elle ne peut exister sans des gens qui la parlent et la comprennent. Nous appellerons « institutions » de telles formes de reproduction du social.

2 Le transfert du pouvoir des individus et des groupes aux institutions entraîne la dépersonnalisation des relations de domination, qui constitue un aspect essentiel du "processus de civilisation ${ }^{1}$ ». La domination, dans les sociétés modernes, est perçue dans la très grande majorité des cas comme un phénomène anonyme. Elle est attribuée à la bureaucratie, à des contraintes économiques objectives, etc. Pour l'histoire de la pensée institutionnelle, cela signifie que la dimension émancipatrice, initialement prévalente, se trouve de plus en plus occultée par la fonction d'assujettissement. Pourtant, les théories de la domination anonyme des structures n'ont pas perdu de vue 
que ces structures sont des faits sociaux, qu'elles ont été créées et maintenues par des sujets humains. La conscience de ce lien imprègne la modernité jusqu'aujourd'hui. Comme nous le verrons à travers une brève histoire de la pensée moderne de l'institution, elle sape la croyance en la permanence des institutions, et mène à la question directrice qui relie les différents textes réunis dans ce numéro : la dimension de l'émancipation se laisse-t-elle incorporer - si paradoxal que cela paraisse - dans la forme institutionnelle d'un pouvoir installé dans la durée?

\section{0 : un prélude révolutionnaire à la conception moderne de l'institution}

Bien que l'origine du mot « institution », comme on sait, prenne sa source dans le droit romain, ce n'est qu'au XIX ${ }^{e}$ siècle que s'impose l'usage actuel du terme. Il découle, en anglais comme en allemand, du verbe français «instituer », qui, depuis déjà la fin du $\mathrm{XII}^{\mathrm{e}}$ siècle, signifiait établir quelque chose de manière durable. Cela incluait en particulier la création de domaines de souveraineté civile et ecclésiastique, ainsi que la fondation d'ordres religieux et juridiques. Cet emploi explique que le verbe et le substantif correspondant («institution») apparaissent dans leur acception traditionnelle au septième chapitre du livre deuxième du Contrat social de Rousseau, où il est question du législateur comme fondateur d'un tel ordre et comme éducateur d'un peuple : «Celui qui ose entreprendre d'instituer un peuple doit se sentir en état, pour ainsi dire, de changer la nature humaine ${ }^{2} »$.

4 Combien l'usage actuel du terme s'écarte de ceux qui sont attestés à l'aube de la Révolution française, c'est ce que l'on peut illustrer sur l'exemple d'un débat tenu à l'Assemblée nationale en janvier 1790. Ce débat, qui allait conduire à la dissolution du Parlement régional de Bretagne, est régulièrement cité, sur la base de l'interprétation présentée par le Trésor de la Langue Française, pour établir que les "institutions" désignaient déjà pendant la Révolution «l'ensemble des structures fondamentales d'organisation sociale ${ }^{3}$ ». En effet, Le Chapelier parle ici des "institutions immuables qui doivent faire le bonheur de tous ${ }^{4}$ ", et qu'il s'agit donc de défendre contre le droit de veto des anciens états. Mais l'expression "institutions immuables» renvoie ici à l'argumentation des conseillers bretons de la Chambre des Vacations ${ }^{5}$ soucieux d'affermir leur position, c'est-à-dire avant tout leur autonomie face à l'Assemblée nationale.

5 Au nom de ces conseillers, le Président de la Chambre des Vacations, La Houssaye, invoquait les droits de souveraineté jadis accordés par traité à la Bretagne, et que les rois de France avaient toujours respectés depuis des siècles. Une grande partie des circonscriptions administratives de Bretagne, disait La Houssaye, avait adressé à la Chambre des cahiers réclamant le maintien de ces droits, qui étaient bien plus que de simples privilèges, contre les prétentions législatives des États généraux, auto-érigés en Assemblée nationale constituante. Car ils fixaient les limites «immuables» qui devaient être opposées au pouvoir législatif de l'Assemblée. À ce titre, les États de Bretagne revendiquaient leur droit à « consentir [...] la loi, l'impôt et tout changement dans l'ordre public de cette province ${ }^{6} »$. Sur quoi Mirabeau s'emporte :

«Ne parlez plus de ces cahiers qui fixent immuablement nos pouvoirs;

immuablement ! Oh! Comme ce mot dévoile le fond de leurs pensées ! [...] Tout est changé, il n'y a plus rien d'immuable que la raison qui changera tout, qui, en 
étendant ses conquêtes, détruira les institutions vicieuses auxquelles les hommes obéissent depuis si longtemps ; il n'y a plus rien d'immuable que la souveraineté du Peuple, l'inviolabilité de ses décrets sanctionnés par son roi $^{7}$. "

Quand donc Le Chapelier parle d'« institutions immuables », il pense certes à l'ordre public, dont les conseillers bretons refusent les transformations, mais pour autant il ne vise nullement - comme pourrait le suggérer la compréhension moderne du terme des organes d'État tels que les ministères ou les services administratifs; il ne fait pas même référence à l'Assemblé nationale elle-même, mais à l'autorité légitime qui habilite celle-ci à édicter des lois et des décrets contraignants (et notamment à suspendre tous les Parlements provinciaux sur le sol français). Il s'agit de défendre cette autorité contre la volonté d'autonomie des Bretons et contre tout corps qui voudrait s'interposer entre l'Assemblée nationale et les citoyens individuels ${ }^{8}$.

6 Malgré la proximité avec les notions d'ordre public et d'intérêt général, les « institutions immuables » de Le Chapelier, tout comme les « institutions vicieuses » de Mirabeau, sont donc avant tout des titres juridiques. L'usage du mot s'insère encore parfaitement dans le spectre sémantique traditionnel, où l'institution désigne le fait d'établir dans une fonction (le plus souvent ecclésiastique, à l'origine), mais aussi l'instruction et l'enseignement (« l'institution des enfants », comme disait Montaigne) ${ }^{9}$.

\section{9/1848 : les institutions révolutionnaires de la liberté}

7 Le mot "institution » fut cependant aussi employé, dès la première année de la Révolution, pour désigner des instances ou des offices nouvellement créés ou renouvelés, tels que les commissions, les tribunaux ou les jurys judiciaires, ou encore les unités militaires et paramilitaires. Il est alors souvent question du sens d'une « institution », ce qui renvoie à l'intention ayant présidé à la fondation d'une telle instance sociale ou à l'attribution de droits spécifiques. Les institutions, ici, ne sont manifestement pas n'importe quelle instance, office ou ordre collectif juridiquement encadré : ce sont avant tout - même lorsqu'elles sont qualifiées d' «immuables ", comme chez Le Chapelier - les instances qui doivent être renouvelées ou créées de toutes pièces au cours du processus révolutionnaire. C'est l'acte de leur instauration qui, par métonymie, permet de désigner comme "institutions " l'instance déjà existante, l'ordre nouvellement établi ou l'office nouvellement créé. Pendant la Révolution, à un moment où les droits et les fonctions du passé n'ont plus cours et doivent être remplacés par d'autres, la dimension sémantique dynamique de l'instauration et de la fondation est donc prépondérante dans l'usage du mot « institution ». Les règles et les ordres anciens ayant été abolis, il est nécessaire d'en introduire de nouveaux. Aujourd'hui, à l'inverse, le terme désigne plutôt les instances, les fonctions et les droits depuis longtemps établis, c'est-à-dire ce à quoi le terme s'était trouvé appliqué seulement par extension pendant l'événement révolutionnaire.

Un coup d'œil dans les dictionnaires allemands montre que le mot «Institution » - dans l'acception spécifique que lui avait donnée la Révolution française, désignant la refondation des structures étatiques - ne se généralisa dans l'aire germanophone qu'après la révolution de 1848. Le Deutsches Rechtswörterbuch, qui décrit la langue juridique jusque vers 1800, ne connaît le mot « Institution » qu'en composition et dans le sens d'« établissement» (d'une charge) et de «bâtiment» (scolaire). Ni le Brockhaus 
Conversations-Lexikon de 1809-1811, ni le Damen Conversations Lexikon de 1834-1838, ne comportent une entrée "Institution». Tandis que le Herders Conversations-Lexikon de 1854-1857 et le Pierer's Universal Lexikon de 1857-1865, avant de citer les usages plus anciens, caractérisent les institutions comme des organismes d'État qui, pour citer le second de ces ouvrages, " doivent garantir les droits et les libertés des citoyens face au gouvernement ». Comme en France, on voit donc s'établir en Allemagne, dans le sillage de l'événement révolutionnaire, une conception moderne de l'institution, désignant les organisations caractéristiques du nouvel ordre public.

\section{0 : Hegel comme source de la conception moderne de l'institution avant la lettre}

Dans le contexte de l'évolution conceptuelle et sociale qui mène à la sémantique actuelle des « institutions ", c'est Georg Wilhelm Friedrich Hegel qui, avec ses Principes de la philosophie du droit, présente pour la première fois l'esquisse d'un ordre moderne à travers lequel la liberté individuelle ne doit pas se trouver seulement garantie, mais véritablement créée. Certes, Hegel ne place pas le concept d'« institution » au centre de ses réflexions ${ }^{10}$. Pourtant, ce que nous entendons aujourd'hui par "institution" correspond largement aux éléments que le philosophe mobilise pour reconstruire $l^{\prime}$ «Esprit objectif ${ }^{11}$ ». On pourrait clarifier ce concept en disant que l'esprit objectif consiste moins dans les idées que les gens ont dans la tête, que dans ce qu'ils sont ${ }^{12}$. C'est la réalité que la liberté de l'esprit acquiert du fait que les humains cherchent à traduire leur volonté en actes. Ces tentatives débouchent sur les ordres de l'agir coordonné, que Hegel discute sous les grandes rubriques du droit, de la moralité et de l'éthicité (ou vie éthique), ce dernier terme renvoyant pour lui aux formes interconnectées de la vie commune: la famille, la société civile et l'État. Dans la philosophie de Hegel, les institutions apparaissent donc dans le contexte de la liberté politique et juridique, ce qui correspond parfaitement à l'esprit révolutionnaire de l'époque.

Mais, comme nous le verrons plus loin, ce n'est pas tant parce qu'elle combine d'une manière révolutionnaire l'institution et la liberté que la philosophie hégélienne marqua de son sceau la conception moderne de l'institution, mais bien plutôt parce qu'elle lui confère un caractère d'objectivité ${ }^{13}$. Déjà, Hegel instaurait entre l'objectivité et la liberté un rapport de tension, sur lequel beaucoup d'interprètes ont insisté. Cette tension amène à se demander si les institutions dans la forme dense programmée par Hegel sont en mesure d'équilibrer adéquatement la fragile relation entre les exigences subjectives de liberté et le pouvoir d'organisation objectif du droit, ou si elles n'aboutissent pas plutôt à maintenir le statu quo historique existant, aux dépens des revendications de liberté individuelle. Dieter Henrich et Axel Honneth, par exemple, à travers leur critique de « l'institutionnalisme fort » de Hegel et des effets possibles de la "surinstitu-tionnalisation » de la vie éthique, expriment de grands doutes quant à la capacité de l'ordre libéral conçu par Hegel à rendre justice au pluralisme moderne des convictions et des projets de $v^{2} e^{14}$. De telles lectures ne traduisent cependant pas seulement la crainte récurrente d'une marginalisation des formes de vie et des routines sociales informelles, mais intersubjectivement partagées : elles signalent aussi que la capacité des institutions sociales à réaliser la liberté est aujourd'hui encore loin de faire l'unanimité. 
11 À l'époque de Hegel, cependant, il semblait naturel de lier la réalisation de la liberté (et des idées en général) à des ordres institutionnalisés, conçus en même temps comme organiques, ainsi que l'atteste l'accord surprenant (compte tenu de tout ce qui les oppose par ailleurs) de la construction hégélienne avec, sur ce point particulier, la théorie concurrente des « institutions du droit » [Rechtsinstitute] de Friedrich Carl von Savigny. Savigny fonda au début du XIXe siècle ce qu'il est convenu d'appeler l'École historique du droit, inspirée principalement par divers courants romantiques dans le champ des sciences juridiques, alors en plein essor. Le droit est envisagé ici comme composante d'une culture globale, au sein de laquelle et conjointement à laquelle il évolue. Cette école se nomme " historique " parce qu'elle cherche, essentiellement à travers la réception du droit romain, à tirer et à développer à partir du système juridique constitué - ou, pour reprendre la formule de Savigny, des convictions formées dans la conscience du peuple -, les normes et les institutions indispensables à la vie et aux orientations normatives des individus ${ }^{15}$. Les règles et les institutions du droit ne peuvent rendre possibles les rapports juridiques, organiser la vie ou aider à résoudre les conflits, que pour autant qu'elles sont l'expression des convictions et des normes qui existent dans une société. Ces matérialisations juridiques des convictions et des normes communes, désignées par l'expression courante de Rechtsinstitute, ne sont cependant pas encore caractérisées par Savigny - conformément à l'historique que nous venons de retracer - comme des institutions (Institutionen) ${ }^{16}$.

\section{1 : Le concept élargi d'institution introduit par la sociologie}

12 En dépit du caractère marginal que conserve le concept à l'époque de Hegel et de Savigny, ces deux auteurs (surtout le premier) marquent la naissance d'une pensée de l'institution avant la lettre, qui allait imprégner la sémantique du terme à partir de la fin du XIX ${ }^{\mathrm{e}}$ siècle. Celle-ci se prolonge au XX $\mathrm{XX}^{\mathrm{e}}$ siècle aussi bien dans la compréhension sociologique du rôle social des institutions que dans la théorie institutionnelle du droit, qui constitua une alternative - certes incontestablement moins influente - aux conceptions dominantes du droit issues du droit naturel et du positivisme juridique.

13 L'émergence de la conception moderne des institutions, inspirée par Hegel, peut se lire à la lumière de l'article «La sociologie, objet et méthode » que Marcel Mauss et Paul Fauconnet rédigèrent pour la Grande Encyclopédie de 1901, et dont nous avons pour cette raison intégré la traduction allemande dans la présente édition. La sociologie y est fondamentalement définie comme une "science des institutions ${ }^{17}$ ». Cette définition montre déjà que la compréhension de ce que sont les institutions s'est à nouveau sensiblement transformée dans la seconde moitié du XIX ${ }^{e}$ siècle, sous l'influence de l'esprit objectif de Hegel. Les institutions ne sont plus seulement les organes destinés à protéger les droits et les libertés des citoyens contre le gouvernement. Elles ne se limitent pas non plus aux "grandes " institutions, c'est-à-dire à ces "personnes juridiques » que Maurice Hauriou caractérisera peu après comme «des institutionspersonnes ou des corps constitués (États, associations, syndicats, etc.) ${ }^{18} »$. Les institutions, pour la sociologie - qui est la science «moderne » par excellence, et pas seulement par sa dénomination -, représentent l'ensemble des pratiques, des rituels, des convictions collectives, les données linguistiques et en général tous les aspects de 
l'organisation sociale, bref: "un ensemble d'actes ou d'idées tout institué que les individus trouvent devant eux et qui s'impose plus ou moins à eux ${ }^{19}$ ".

Si la parenté d'une telle conception de l'institution avec «l'esprit objectif» de Hegel, qui embrasse lui aussi tout un éventail de rapports formels, semi-formels ou tout à fait informels est frappante - quoique pas complètement surprenante chez des élèves de Durkheim, qui était lui-même familier de l'œuvre hégélienne -, elle présente cependant un changement d'accent décisif par rapport, notamment, aux Principes de la philosophie $d u$ droit. La référence à la liberté humaine, centrale pour Hegel, se trouve en effet totalement occultée ici par le caractère socialement déterminé de l'institution. Ce qui à l'ère des révolutions bourgeoises était une réalité à façonner, disponible, est devenu pour la sociologie du $\mathrm{XX}^{\mathrm{e}}$ siècle une réalité donnée d'avance, qui produit et garantit les régularités de la vie sociale. Dans son ouvrage Urmensch und Spätkultur [L'homme primitif et la civilisation tardive] Arnold Gehlen parle même dans ce contexte d'un «a priori acquis ${ }^{20} "$, terme qu'on peut certainement considérer comme un précurseur du concept d'«a priori historique » que Michel Foucault allait forger avec succès dans les années $1960^{21}$. Dans certaines théories sociologiques du XXe siècle, dont l'influence s'est maintenue jusqu'à aujourd'hui, c'est à travers les institutions - comprises en ce sens large, incluant les formes d'action, de perception et d'interprétation - que s'opère la " construction sociale de la réalité2 ${ }^{2}$ ".

Ce déplacement sémantique du terme d'« institution » - qui désignait initialement une entité susceptible d'être fondée (notamment en raison) et modifiée, et qui renvoie pour finir à un pur donné, précédant l'expression de toute volonté individuelle et conditionnant l'être des individus - correspond à un déplacement de la réalité sociale. Après les bouleversements des révolutions bourgeoises, le concept d'institution mobilisé par la sociologie porte l'empreinte de la consolidation de la nouvelle forme de société. L'expérience d'une transformation toujours possible se résorbe progressivement dans les pratiques de la reproduction sociale. Alors que la possibilité des révolutions - y compris de révolutions d'un type nouveau, qui pourraient succéder à une révolution bourgeoise - demeurait, en tant que possibilité, hors de doute jusque tard dans le $\mathrm{XIX}^{\mathrm{e}}$ siècle, les théories sociologiques de l'institution du $\mathrm{XX}^{\mathrm{e}}$ siècle cherchent au contraire à expliquer pourquoi un changement radical de la structure sociale est improbable.

\section{9 : Penser la possibilité de l'effondrement institutionnel}

16 L'actualisation par Gehlen de la conception sociologique de l'institution - malgré les nombreuses références qu'il fait lui aussi à l'esprit objectif de Hegel - n'est cependant pas sans nuances, précisément en ce qui concerne le passage d'une conception révolutionnaire de l'institution, tournée vers la refondation du réel, à une simple description de la reproduction sociale. Chez lui s'exprime en effet un pessimisme culturel qui constate la perte de stabilité des institutions, du moins sous leurs formes connues $^{23}$, et offre ainsi un contrepoids à l'analyse typique du discours institutionnel, au centre de laquelle se trouve la stabilisation des attentes comportementales. La déstabilisation des institutions constitue cependant selon Gehlen un processus extrêmement complexe et nullement linéaire. Ainsi, l'introduction du monothéisme aurait déjà eu pour conséquence que « les institutions ont depuis lors perdu leur pouvoir 
proprement théogonique, leur capacité à créer des dieux ${ }^{24} »$. La question est désormais de savoir ce qui pourrait garantir en dernière instance la validité des institutions terrestres, après qu'elles ont perdu leur statut sacral, intangible.

Pour Gehlen la stricte séparation introduite par le monothéisme entre l'au-delà et le monde d'ici-bas détermine l'apparition des sciences profanes de la nature, avec tout ce qui en découle en termes de domination technique des conditions d'existence, parce qu'elles excluent toute intervention intramondaine de démons et d'autres êtres de cette sorte. Mais, d'un autre côté, cette séparation constitue aussi le point de départ d'une évolution qui mène à l'intériorisation et au triomphe du subjectivisme. Les sciences de la nature et le subjectivisme vont la main dans la main, estime Gehlen, quand aujourd'hui la conformité comportementale des membres de la société - qui ne peut reposer sur de simples convictions, mais suppose nécessairement des institutions - se trouve rapportée à des contraintes matérielles. Aux questions de sens touchant les institutions il n'est alors plus possible de répondre qu'en recourant à des convictions subjectives.

«La disposition fonctionnalisée à se soumettre se transmet alors à la société économique et politique tout entière, et l'on n'hésite pas à en abuser. Au revers se dessine la révolte non seulement de la personne, mais de la pure et simple subjectivité25.»

Malgré le «manque de moyens » que Gehlen attribue d'abord à cette révolte de la subjectivité, elle constitue en fin de compte, à ses yeux, la pire menace pour les institutions, car cette

«forme de conscience imposée par le système culturel a permis de poursuivre la destruction des institutions existantes non plus par une action ouverte, organisée, mais sans bruit, de l'intérieur, par un effet émollient qui n'est même pas toujours volontaire [...] La force d'inhibition des idées directrices se relâche et la critique se trouve libérée ${ }^{26} . »$

Autrement dit, ce sont l'individualisation et la critique de la société qui menacent aux yeux de Gehlen la transcendance des institutions, leur intangible sacralité.

19 La victoire de la Révolution d'Octobre en Russie, l'avènement du national-socialisme en Allemagne, tout comme (selon cette tradition de pensée) la défaite des puissances de l'Axe dans la Seconde Guerre mondiale, contribuèrent sans doute à ébranler la foi, née de la récente consolidation des ordres bourgeois, en la stabilité à toute épreuve des institutions. C'est en ce sens que Helmut Schelsky évoque dans une conférence prononcée au début de 1949 la nécessité d'analyser la durabilité des institutions : «Il fallait peut-être que nous fassions l'expérience de l'instabilité de notre propre environnement social, de l'effondrement ou du moins de la fragilisation de presque toutes nos anciennes institutions, pour que cette question devienne un besoin général de la pensée ${ }^{27}$. "

20 À la différence de Gehlen, toutefois, Schelsky ne s'installe pas dans le pessimisme culturel. Sa réflexion sur la stabilité des institutions s'efforce de tenir compte du fait que la critique de la société est une réalité qui doit être intégrée dans une théorie aboutie des institutions. Il écrit ainsi en 1949: «La possibilité d'un contrôle analytique autocritique fait aujourd'hui partie des exigences de base d'une institution stable, non moins qu'une conscience fortement motivée de son programme et de son ancrage dans le droit $^{28}$. » À cet égard, la réflexion schelskyenne sur la durabilité des institutions ne renvoie pas à une théorie du déclin des institutions, mais vise à construire une théorie du changement institutionnel contrôlé, articulé aux besoins sociaux et à leur évolution. 


\section{8 : l'exigence paradoxale de la modernité}

21 En exigeant d'intégrer la critique de la société dans la conception même des institutions, il se démarque également de ces théories du changement social qui comprennent la modernité comme un système évolutif de différenciation sociale. Ainsi, au milieu des années 1960, Niklas Luhmann - pour prendre un exemple éminent envisage les institutions exclusivement comme des «attentes comportementales généralisées dans le temps, dans leur contenu et dans leur champ d'application sociale $^{29}$ ", et dont la différenciation en systèmes fonctionnels interconnectés serait menacée par la subjectivation déjà dénoncée chez Gehlen. Cela signifie que pour Luhmann aussi les sociétés modernes, en admettant la possibilité, essentielle pour elles, d'une critique des attentes comportementales stabilisées ne font rien d'autre que saper leurs propres fondations. Cette menace de «dé-différenciation» immanente au système politique moderne ne doit cependant pas, selon Luhmann, être neutralisée par un refoulement de la politique, mais circonscrite par une catégorie spécifique d'institutions : les droits fondamentaux. Les droits fondamentaux visent à garantir que l'action menée par l'État en vue de ses propres objectifs politiques «doit toujours présupposer dans l'ordre social d'autres modes de réalisation, d'autres systèmes permettant aux acteurs de poursuivre leurs intérêts, d'autres sources de pouvoir et de prestige social ${ }^{30} \%$. Autrement dit, l'action politique est soumise à l'attente comportementale du respect d'autres sphères institutionnelles et de leur logique propre. Mais alors elle ne peut plus intervenir dans la constitution de ces sphères, ce qui prive la critique des institutions de son aiguillon.

Schelsky, en revanche, ne refoule pas le problème de la stabilisation et de la critique dans des sphères sociales séparées. Dans une contribution au débat sur la théorie de l'institution, publiée en 1970, il résume la question du changement institutionnel alors certainement ravivée par les controverses engagées autour de 1968 - au moyen d'une description paradoxale des institutions modernes, qui s'oppose désormais explicitement aux théories gehleniennes de la décadence. Schelsky établit ici que

« les besoins de la subjectivité moderne migrent dans les institutions avant tout comme besoins d'expression de l'individu, et cherchent à y trouver leur satisfaction. [...] Les revendications critiques de cette subjectivité réfléchissante, dirigées contre les "contraintes", c'est-à-dire contre les dimensions fonctionnelles et objectives de chaque institution, ne visent pourtant pas à transformer directement l'institution dans sa réalité concrète ou dans son esprit général : cette réflexion critique et son expression veulent plutôt s'établir avant tout dans la durée, elles veulent être intégrées dans l'institution [mitinstitutionalisiert ${ }^{31}$. »

Ici, Schelsky s'écarte manifestement de l'acception large du concept sociologique d'institution, lequel inclut déjà certaines formes simples de perception, de jugement et d'action. Les institutions sont pour lui ces formes de comportement collectif stabilisé dont l'organisation est portée par des techniques et un mode de communication spécifiques. Dans sa caractérisation de l'institution moderne, nous retrouvons en outre l'exigence - déjà déterminante dans les sources révolutionnaires de la pensée de l'institution, et plus tard chez Hegel - selon laquelle les institutions doivent être les formes sociales de réalisation de la liberté individuelle. 

les actions des individus et stabiliser efficacement leurs rapports de coopération, sans pour autant réprimer ou manipuler la subjectivité individuelle: elles doivent, au contraire, rester ouvertes aux revendications de celle-ci. Une problématique analogue caractérise déjà la description hégélienne de l'héritage libéral de la Révolution française ${ }^{32}$. Schelsky croit cependant que l'exigence paradoxale adressée par la modernité aux institutions pourrait être atténuée par le fait que les institutions modernes, si elles admettent l'expression de la subjectivité (critique), n'en admettent que l'expression et rejettent fondamentalement toute modification factuelle qui découlerait «directement, sur le plan fonctionnel ou politique ", de cette critique, parce que «toute modification concrète ou politique de l'institution par l'effet direct d'une subjectivité critique, dissout en retour celle-ci et la traduit en termes de fonctionnalité et de domination ${ }^{33}$ ».

\section{3 : une protection institutionnelle de la critique non institutionnalisable?}

Treize ans après que Schelsky eut formulé l'exigence paradoxale adressée aux institutions modernes - treize ans qui, en Europe de l'Ouest, furent marqués par la montée de nouveaux mouvements sociaux, et durant lesquels Cornelius Castoriadis s'efforça de réinscrire le moment révolutionnaire de l'«instauration» dans la théorie $\mathrm{du}$ concept sociologique large d'institution ${ }^{34}$, sans pourtant fournir un critère permettant de dire à quel moment une institution moderne au sens étroit du terme intègre effectivement la conscience de sa transformabilité -, treize ans plus tard, donc, il était clair qu'on ne viendrait pas si facilement à bout du paradoxe. C'est pourquoi Jürgen Habermas, à l'occasion d'une réflexion sur la désobéissance civile, chercha à le résoudre à travers une double requête.

Celle-ci s'adressait d'une part aux citoyennes et aux citoyens désireux d'exercer leur droit de résistance face à des "violations légales de la légitimité ${ }^{35}$ ": "Celui qui enfreint les règles doit scrupuleusement examiner si le choix de moyens spectaculaires est réellement adapté à la situation, ou s'il ne résulte pas simplement d'une posture élitiste ou d'un élan narcissique, c'est-à-dire d'un geste de présomption ${ }^{36}$.» Mais la demande s'adressait également, d'autre part, aux institutions, et notamment à l'État, avec son pouvoir juridique de sanction visant paradoxalement à "protéger et à entretenir la méfiance envers une injustice présentée sous des formes légales, bien que cette méfiance ne puisse pas trouver une expression institutionnellement garantie ${ }^{37}$ ». Dans ses deux versants, une telle requête reste cependant chez Habermas une simple exhortation - même si elle s'appuie au plan moral sur les expériences du nationalsocialisme -, à laquelle même un débat ultérieur sur la théorie des institutions politiques et de leur transformation n'a pas permis de donner corps ${ }^{38}$.

Schelsky et Habermas se rejoignent néanmoins en ce qu'ils cherchent tous deux à résoudre le paradoxe sur l'arrière-plan d'un ordre juridique, plus précisément: constitutionnel, censé fournir le cadre d'une transformation contrôlée par les institutions. Chez Habermas, ce cadre comprend, comme nous venons de le voir, les espaces moins strictement réglementés de la discussion publique, dans lesquels la critique peut s'exprimer et l'opinion se former librement. La dépendance des institutions à l'égard du droit, déjà postulée par Gehlen, est cependant maintenue chez 
Habermas, de sorte que le paradoxe risque de se trouver à nouveau vidé de son contenu par une solution à la Luhmann, avec un dispositif institutionnel garanti sur le plan des droits fondamentaux. Mais par cette inscription du paradoxe dans le cadre d'un ordre constitutionnel juridiquement défini, le débat se rapproche à nouveau du schéma hégélien, où l'institutionnalisation réussie de la liberté est nécessairement pensée, en fin de compte, comme un ordre juridique. Aussi n'est-il pas étonnant de voir des éléments de la théorie institutionnelle du droit, elle aussi inspirée de Hegel, réapparaître régulièrement dans la théorie sociologique des institutions.

\section{5 : La théorie institutionnelle du droit}

La théorie institutionnelle $\mathrm{du}$ droit s'oppose aux théories traditionnellement dominantes sur deux points essentiels :

1) relativement au rôle paradigmatique de la nature, qui se retrouve aussi bien dans l'Antiquité, avec la distinction nomos/physis (loi/nature), que dans la pensée moderne, lorsque le droit naturel se fonde sur une nature sacrée et/ou humaine ;

2) relativement, en particulier, à la croyance au caractère fondamental des droits naturels subjectifs (natural rights), c'est-à-dire de droits «naturels et inaliénables » de l'être humain individuel - une idée dont l'origine se trouve chez Hobbes (mais qui s'annonce déjà chez Suarez et Grotius).

Pour la théorie institutionnelle du droit, celui-ci n'est justement pas borné par de telles données naturelles, pas plus qu'il ne découle de données immuables - qu'elles soient comprises comme des lois de la nature ou comme des droits naturels. La théorie institutionnelle envisage au contraire les ordres institutionnels d'une société comme l'arrière-plan sans lequel ne peuvent être compris les lois et les droits, ni même le type de subjectivité juridique spécifique qui leur est associé. Par là, la théorie institutionnelle du droit ne conteste pas seulement les théories qui s'inscrivent dans la tradition du droit naturel. Elle contredit également le positivisme juridique, qui remplaça au cours du XIX siècle le droit naturel comme conception dominante du droit. Le positivisme juridique s'appuie, en gros, sur l'idée que le droit positif se suffit à lui-même et rend inutile tout recours à un fondement «naturel » du droit. Dans la variante la plus répandue au XIX ${ }^{\mathrm{e}}$ siècle (c'est-à-dire avant que l'histoire de la théorie vienne à se compliquer), c'est la volonté souveraine du législateur (ou en dernière instance de l'organe constituant) qui est mobilisée pour remplacer la nature (divine ou humaine) et fonder ainsi la validité du droit positif ${ }^{39}$. C'est justement cette volonté souveraine que la théorie institutionnelle du droit va maintenant considérer comme un effet, et non plus comme un factum brutum préalable.

Cela dit, la théorie institutionnelle du droit ne constitue pas une doctrine unifiée $e^{40}$. On peut schématiquement distinguer entre les théories de la première moitié $\mathrm{du} \mathrm{XX}$ siècle (Maurice Hauriou, Santi Romano et Carl Schmitt) et les théories institutionnelles «actuelles ». Ces dernières s'inscrivent dans le courant analytique de la philosophie et donnent une importance considérable à la philosophie du langage. La théorie des actes de langage de John Austin, reprise et prolongée par John Searle, propose en effet une approche des « faits institutionnels » sur laquelle des philosophes du droit comme John McCormick ou Joseph Raz s'appuient aujourd'hui. Malgré toutes leurs différences, les théories institutionnelles classiques et actuelles ont en commun de rejeter l'antithèse habituelle entre droit naturel et positivisme juridique. Car, si elles n'ont rien de 
naturel, les institutions en général (pas seulement les instituions juridiques) acquièrent néanmoins ce que Hegel, à la suite d'Aristote, appelait un caractère de "seconde nature ». Elles sont en quelque sorte des réalités culturelles devenues naturelles. Leur existence apparait aux individus comme une évidence, et elle est en ce sens "naturelle", préalable à toute action rationnelle. Mais en reconnaissant aux institutions un tel primat méthodologique, la théorie institutionnelle du droit intègre à son tour ce qui constitue l'élément central des théories sociologiques des institutions.

C'est tout à fait dans le sens d'une concordance entre les théories juridiques et sociologiques qu'Hauriou, dans ses textes publiés au tout début du XX $\mathrm{XX}^{\mathrm{e}}$ siècle ${ }^{41}$, affirme que l'institution est la réalisation d'une "idée-mère ${ }^{42}$ ", à laquelle elle confère une "existence objective ${ }^{43}$ ", une "vie propre et autonome ${ }^{44}$ ». Mais la théorie institutionnelle d'Hauriou va encore plus loin dans l'affirmation du caractère naturel de l'idée. La réalisation de l'idée fondamentale spécifique à chaque institution montre selon lui la «nature objective» que cette idée aurait «dès le début ${ }^{45}$ ». Les idées constituent une sorte d' d $^{\text {âme objective }}{ }^{46}$ » qui se reflèterait dans chaque individu. « Les idées objectives existent d'avance dans le vaste monde, incorporées aux choses qui nous entourent ${ }^{47}$. " L'institution est en quelque sorte une idée objectivée, une quasinature, qui s'offre aux individus comme un cadre d'action évident.

La théorie de l'institution développée par Hauriou, y compris la «fondation continuée ${ }^{48}$ » qu'il décrit dans ces pages, pourraient donc effectivement ramener aux problèmes du droit naturel (comme l'explique Olivier Beaud dans le texte que nous publions dans ce numéro). Mais, selon dans une autre lecture, la théorie des institutions développée par Hauriou pourrait aussi - nolens volens - contribuer à dégager une troisième voie entre droit naturel et positivisme juridique.

Pour la discussion allemande, dans laquelle Hauriou fut d'abord mobilisé par Carl Schmitt, puis, à sa suite, par Gehlen et Schelsky, c'est justement cette troisième voie qui se révéla déterminante. La construction conceptuelle de l'«idée directrice», au premier chef, fut intégrée, soit directement sous sa forme française, soit traduite en allemand par le terme "Leitidee». Chez Hauriou lui-même, cette idée doit être « objective » de la manière que nous avons dite. En effet, elle ne désigne pas le but ou la fonction d'une entreprise collaborative, à laquelle les individus pourraient avoir un intérêt partagé sur un plan purement extérieur, elle est l'objet même de l'action commune, ce qui définit l'action commune comme action. Pour expliquer la différence entre un tel « objet » et la fonction ou le but de l'activité, Hauriou prend l'exemple de l'entreprise économique organisée en société commerciale. Si le but d'une telle société est le profit, sa fonction la production ou la distribution de biens ou de services, son idée directrice est, dit-il, la spéculation, - et peu importe pour la poursuite de son existence quelle sorte d'activité particulière on y mène, pourvu que la spéculation se poursuive ${ }^{49}$. Une entreprise peut élargir ses domaines d'activités, les déplacer partiellement ou en totalité, et pourtant continuer à exister en tant que société. À la différence du but (dans notre exemple: le profit), l'idée directrice est plus déterminante pour l'existence de l'entreprise, car elle définit aussi la manière dont ce but abstrait est poursuivi (en l'occurrence : par l'achat et la vente de marchandises) sans entrer toutefois dans le détail particulier de la démarche.

L'« idée directrice» d'Hauriou ne peut être identifiée que par une analogie très grossière à l'idée hégélienne d'esprit objectif. Certes, celle-ci ne trouve elle aussi sa réalisation que dans l'ordre institutionnel moderne, mais les idées directrices 
d'Hauriou se rapportent d'une manière beaucoup plus concrète à des contextes d'action déjà existants, dans la mesure où elles visent à éclairer la coopération générée, non pas par la société tout entière, mais par les institutions particulières que celle-ci renferme. Ainsi Gehlen et Schelsky s'intéressent aux idées directrices d'Hauriou parce qu'elles sont objectives, mais aussi périssables. Tout comme les « institutions éternelles » de Le Chapelier, elles prennent volontiers pour les membres d'une société le caractère d'évidences incontournables; pourtant elles sont conditionnées par leur époque, elles présentent une dimension historique. La tension entre la durabilité et la variabilité, entre le caractère d'évidence - sur lequel le droit naturel fait fond - et la nécessité pour elles d'être établies et maintenues en vie - sur laquelle insiste le positivisme juridique -, cette tension est donc d'emblée inscrite dans les institutions. Elles doivent se transformer au fil du temps, et peuvent fort bien disparaître lorsqu'elles perdent leur objet.

\section{0 : continuer à travailler sur le paradoxe}

La troisième voie entre le droit naturel et le positivisme juridique, telle que suggérée par une théorie institutionnelle du droit, ne suffit pas, à elle seule, à résoudre l'exigence paradoxale que la modernité adresse aux arrangements institutionnels. Si l'on veut que «l'idée directrice » soit davantage qu'une brique de plus dans l'édification d'un pessimisme culturel à la Gehlen, qui n'y voit qu'une répétition générale de l'effondrement de toute orientation collective dans une modernité vouée à la réflexivité, alors elle doit être pensée dans l'interaction entre la matérialisation institutionnelle et l'interprétation qui l'accompagne. Dans un tel jeu d'interaction, l'idée et sa matérialisation s'engendrent et s'étayent l'une l'autre. Mais pour les questions qui concernent la critique et la variabilité des institutions, cela signifie justement qu'il ne peut de facto exister d'institutions dont l'idée directrice n'admette aucune modification. Le pouvoir condensé dans les institutions ne peut être neutralisé sur le seul plan des idées. Mais il est également limité dans son évolution, quand il est reste soumis à une même conception $d u$ but et du caractère de l'interaction institutionnalisée. Une société de commerce peut changer de forme et restructurer ses activités, tant qu'elle se conforme à l'idée de "spéculation ", c'est-à-dire à la recherche de profit, les impératifs économiques continueront à dicter son modèle de coopération - si dysfonctionnel et nuisible celui-ci puisse-t-il être, à maints égards, pour la vie en commun. Pourrait-on à l'inverse dire qu'une institution porte consciemment inscrite en elle la possibilité de changer à tous les niveaux, y compris dans son idée directrice encore non réalisée, sans mettre en péril sa capacité même à structurer l'ensemble de la vie sociale?

La généalogie et l'histoire de la pensée institutionnelle, telles que nous les avons brièvement retracées ici, ne font apparaître ni dans les théories sociologiques ni dans les théories juridiques une réponse satisfaisante à cette exigence paradoxale adressée à l'institution moderne, bien que ces théories soulignent tantôt l'un, tantôt l'autre versant du paradoxe. Si les aspects révolutionnaires de la refondation et de l'instauration intentionnelle dominaient initialement le discours sur les institutions, ce fut plus tard le thème de leur inertie qui passa au premier plan. Le concept d'institution ne réagit à la liquéfaction moderne du social, comme nous avons pu le voir chez Schelsky et Habermas, qu'en rendant explicite l'exigence paradoxale adressée aux 
formes d'organisation de la société. Les institutions doivent expressément rendre la vie sociale possible en introduisant dans les relations et les interactions sociales un facteur de stabilité, sans lequel aucune attente comportementale, et donc aucune coordination efficace des actions ne seraient possibles. Mais en même temps leur concept doit non moins expressément se présenter comme un produit de la coopération humaine, de sorte que leur pouvoir stabilisateur aille toujours de pair - c'est l'exigence qui leur est implicitement adressée - avec leur propre historicité. Les institutions doivent être stables et en même temps créer les conditions de leur propre transformation.

Si nous avons souhaité dans la présente publication reprendre la discussion francoallemande sur le concept moderne d'institution, c'est en référence explicite à l'historicité des institutions, laquelle s'inscrit dans le contexte de la conviction moderne selon laquelle les conditions de la coexistence politique et, plus généralement, sociale sont toujours le résultat de l'agir humain. Une telle conviction implique que ces conditions «faites " par l'homme peuvent également être changées par l'homme. Nous situons donc délibérément la discussion du concept que la théorie sociale forge de l'institution dans le cadre d'un des problèmes fondamentaux de la modernité, portant sur la manière dont le savoir concernant la signification décisive de l'agir humain pour les ordres de la coexistence humaine peut trouver un écho adéquat dans ces ordres eux-mêmes.

Bien entendu, aucun des textes réunis ici ne résoudra, à lui seul, le problème évoqué. Mais dans leur jeu d'interaction, ils renvoient tous à des ressources présentes dans l'histoire des idées et à des approches nouvelles, offrant des perspectives prometteuses au projet de repenser le problème à nouveaux frais.

Olivier Beaud, par exemple, ne se contente pas dans l’article déjà cité («Hauriou et le droit naturel») de retracer les différents paliers de l'évolution du concept d'«institution" chez Hauriou. Il montre aussi comment celui-ci tire d'anciennes conceptions de philosophie morale l'idée selon laquelle les institutions, bien qu'elles visent à la stabilité, restent toujours accessibles aux exigences morales, et doivent pour cette raison - conclut l'auteur - présenter des capacités d'adaptation et d'amélioration. Le texte suivant, de Vincent Descombes, est tiré de son livre Les Institutions du sens. Descombes prend appui sur la question d'Hauriou quant à ce qui fait l'objectivité des idées sur lesquelles s'appuient les institutions. Il mobilise, pour expliciter et actualiser «l'esprit objectif » de Hegel, les usages anglo-saxons et français du concept élargi d'« institution », tel qu'il est utilisé en sociologie. Descombes ouvre ainsi la discussion aux influences de la philosophie sociale actuelle. À cet égard, ses réflexions constituent aussi un pont avec le premier texte allemand de notre ensemble, l'article de Rahel Jaeggi, « Qu'est-ce qu'une (bonne) institution? ». Jaeggi traduit l'exigence de stabilité adressée aux institutions en la rapportant à leur capacité spécifique à résoudre des problèmes. L'exigence de transformabilité des institutions peut dès lors se comprendre comme une exigence d'adaptation concrète et non arbitraire. Sa thèse est que les institutions sclérosées sont celles qui ont perdu leur capacité à résoudre un problème spécifique. La perte de cette fonction constitue aussi l'arrière-plan de la thèse du "déclin de l'institution" défendue par François Dubet dans son article "Déclin de l'institution et/ou néolibéralisme ». Dubet y expose cette thèse en prenant l'exemple de l'institution scolaire, et établit que le déclin des institutions peut très bien être une conséquence de leur succès - en l'occurrence la réalisation des objectifs de la formation scolaire. Dans son texte «La "morale" des institutions ", M. Rainer Lepsius posait déjà 
la question que soulèvent sur un plan systématique les approches de Jaeggi et Dubet : il s'interrogeait, dans le contexte de l'effondrement de la République démocratique allemande en 1989, sur les conditions qui déterminent la capacité d'adaptation des institutions ${ }^{50}$, montrant qu'elles ne peuvent être contrôlées et donc transformées dans leur fonctionnement que si, contrairement à ce qui se passe dans les totalitarismes, leurs exigences de validité se trouvent limitées par d'autres institutions. À la différence de Jaeggi et Dubet, qui argumentent à partir d'institutions particulières, Lepsius n'envisageait de solution au problème des institutions que dans l'interaction entre diverses logiques institutionnelles. Comment pourrait s'effectuer un tel jeu d'interaction, c'est ce qu'Isabell Hensel et Gunther Teubner exposent dans « Matrix reloaded ", à partir d'une analyse des influences économiques sur la science médicale. Mais dans la mesure où Hensel et Teubner abordent la concurrence des logiques institutionnelles comme un conflit concernant les droits fondamentaux, ils mettent implicitement le doigt sur une difficulté inhérente à l'approche de Lepsius. La relativisation d'une logique institutionnelle, si elle n'est pas régulée par le droit, peut aussi agir comme une dangereuse subversion ou, pour reprendre une expression de Jürgen Habermas, comme une forme de " colonisation ». La piste, suggérée par Hensel et Teubner, d'une juridicisation des conflits interinstitutionnels se trouve à son tour mise en question dans l'article de Sabine Hark, «Travers.es du droit ». Hark identifie, relativement aux modes d'existence marginalisés, deux manières d'invoquer le droit. Elle caractérise l'une comme "désinstitutionnalisation", ce qui doit se comprendre comme un moyen de protéger de tels modes d'existence contre les exigences institutionnelles venues de l'extérieur. L'autre manière aspire à la reconnaissance et à la confirmation par les institutions dominantes. Cette deuxième approche semble d'abord séduisante, parce qu'elle supprime ou du moins amortit les "coûts" personnels de la déviance (déjà thématisés chez Lepsius). Hark signale cependant que cette option entraîne un renforcement des institutions dominantes (par exemple le mariage) et sape ainsi la revendication de leur transformabilité. Contre une aussi forte exigence de transformation institutionnelle, on est tenté de mobiliser des arguments qui, reprenant l'analyse de Jaeggi, renvoient à la nature des problèmes que les institutions ont à maîtriser. Il semble donc indiqué de revenir, avec "L'institution juridique de la nature » de Yan Thomas, sur la question du droit naturel, si importante pour la discussion française depuis Hauriou. De fait, dans cette étude hautement érudite sur le droit romain, Thomas met en évidence les espaces de liberté et la créativité dont jouit le droit dans sa relation avec la nature. En analysant minutieusement les textes dans lesquels l'on cherche souvent une présentation du "droit naturel ancien ", il établit que la nature à laquelle se réfère le discours juridique est une nature instituée par le droit comme sa propre limite, une nature institutionnalisée, qui n'a d'autre rôle que de rappeler le néant normatif qui limite et enveloppe le droit lui-même. La nature, nous dit Thomas, n'est donc pas une «norme ultime ", elle n'est pas " une loi qui dicte les fondements du droit », elle est elle-même une institution au sens d'une chose instituée. Elle est instituta, et non instituans. Et lorsqu'elle intervient effectivement dans l'argumentation juridique, c'est à la manière d'une fiction permettant, sur le mode du "comme si », de résoudre des cas pour lesquels le droit civil positif ne propose aucun outil : loin d'être fondateur et immuable, le droit naturel est flexible.

40 Mais si la voie d'un « retour à la nature » est fermée, puisque la nature est toujours déjà un effet de l'institutionnalisation, il ne nous reste plus qu'à comprendre la «seconde 
nature» des institutions comme notre œuvre commune, et donc aussi comme l'expression de notre savoir en mouvement et de notre critique collective, dont nous avons encore à maîtriser les effets en retour sur nous-mêmes.

\section{BIBLIOGRAPHIE}

Berger, P. L. / Luckmann, T. (2018 [1966]) : La construction sociale de la réalité, trad. par P. Taminiaux, Paris, A. Colin ;

orig. : The Social Construction of Reality : a Treatise in the Sociology of Knowledge (1966), London, Penguin Books, 1991.

Castoriadis, C. (1975) : L'Institution imaginaire de la société, Paris, Éditions du Seuil.

Dubiel, H. (1976) : Article «Institution », in : Ritter, J. / Gründer, K. (éd.) : Historisches Wörterbuch der Philosophie, t. 4, Bâle : Schwabe, col. 418-424.

Elias, N. (1976 [1939]) : Über den Prozess der Zivilisation, 2 vol., Francfort-sur-le-Main, Suhrkamp ; traduit en français en deux parties : La Civilisation des mœurs et La Dynamique de l'Occident, trad. par P. Kamnitzer, Paris, Calmann-Lévy, 1973 et 1975.

Fauconnet, P. / Mauss, M. (1901) : « La sociologie : objet et méthode », in : La Grande Encyclopédie, vol. 30, Paris, Société anonyme de la Grande Encyclopédie, p. 165-176 ; rééd. in : Mauss, M. (1968) : Essais de sociologie, Paris, Minuit (coll. « Points Essais »), p. 6-41.

Foucault, M. (1966) : Les Mots et les choses, Paris, Gallimard.

Foucault, M. (1970) : L'Archéologie du savoir, Paris, Gallimard.

Gehlen, A. (1986) : Urmensch und Spätkultur, $5^{\mathrm{e}}$ éd., Wiesbaden, Aula.

Göhler, G. (éd.) (1987) : Grundfragen der Theorie politischer Institutionen. Forschungsstand - Probleme Perspektiven, Opladen, Westdeutscher Verlag.

Göhler, G. (éd.) (1997) : Institutionenwandel, Opladen, Westdeutscher Verlag.

Habermas, J. (1985) : « Ziviler Ungehorsam. Testfall für den demokratischen Rechtsstaat » (1983), in : id. : Die Neue Unübersichtlichkeit, Francfort-sur-le-Main, Suhrkamp, p. 79-99.

Haferkamp, H.-P. (2018) : Die Historische Rechtsschule, Francfort-sur-le-Main, Klostermann.

Han, B. (2003) : « L'a priori historique selon Michel Foucault : difficultés archéologiques », in : Da

Silva, E. (éd.) : Lectures de Michel Foucault, II : Foucault et la philosophie, Lyon, ENS Éditions, p. 23-38.

Hauriou, M. (1921) : Précis de droit administratif et de droit public à l'usage des étudiants en licence (2e et 3 e années) et en doctorat ès-sciences politiques, $10^{\circ}$ éd., Paris, Sirey.

Hauriou, M. (1933 [1925]) : « La théorie de l'institution et de la fondation (essai de vitalisme social) ", in : id. : Aux sources du droit : le pouvoir, l'ordre et la liberté, Paris, Librairie Bloud \& Gay ( Cahiers de la nouvelle journée », n²3), p. 89-128.

Hauriou, M. (1933 [1928]) : « Le pouvoir, l'ordre, la liberté et les erreurs des systèmes objectivistes ", in : id. : Aux sources du droit : le pouvoir, l'ordre et la liberté, Paris, Librairie Bloud \& Gay («Cahiers de la nouvelle journée », n²3), p. 72-86. 
Hauriou, M. (1965 [1925]) : « Die Theorie der Institution und der Gründung », in : id. : Die Theorie der Institution und zwei andere Aufsätze von Maurice Hauriou, éd. par Roman Schnur, trad. par Hans et Jutta Jecht, Berlin : Duncker \& Humblot, p. 27-66.

Hegel, G. W. F. (2013) : Principes de la philosophie du droit, trad. par J.-F. Kervégan, Paris, PUF.

Hegel, G. W. F. (1988) : Encyclopédie des sciences philosophiques, trad. par B. Bourgeois, Paris, Vrin.

Hegel, G. W. F. (1963) : Leçons sur la philosophie de l'histoire, trad. par J. Gibelin, Paris, Vrin.

Henrich, D. (1983) : «Einleitung des Herausgebers », in : G. W. F. Hegel : Philosophie des Rechts. Die Vorlesung von 1819/20 in einer Nachschrift, Francfort-sur-le-Main, Suhrkamp, p. 9-39.

Honneth, A. (2008) : Les Pathologies de la liberté: une réactualisation de la Philosophie du droit, trad. F. Fischbach, Paris, La Découverte.

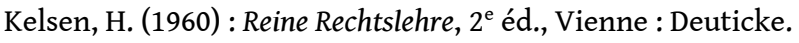

Luhmann, N. (2019) : Grundrechte als Institution. Ein Beitrag zur politischen Soziologie, $6^{\mathrm{e}}$ éd., Berlin : Duncker \& Humblot.

Montaigne, M. de (1962 [1850]) : Essais, Paris, Garnier.

Rousseau, J.-J. (1964 [1762]) : Du contrat social ou Principes du droit politique, in : CEuvres complètes, vol. III, Paris, Gallimard, p. 347-470.

Schelsky, H. (1965) : « Über die Stabilität von Institutionen, besonders Verfassungen.

Kulturanthropologische Gedanken zu einem rechtssoziologischen Thema », in : id. : Auf der Suche nach Wirklichkeit, Düsseldorf / Cologne : Eugen Diedrichs Verlag, p. 33-55.

Schelsky, H. (1970) : « Zur soziologischen Theorie der Institution », in : id. (éd.) : Zur Theorie der Institution, Düsseldorf : Bertelsmann Universitätsverlag (Interdisziplinäre Studien, t. 1), p. 9-26.

Simitis, S. (1989) : «Die Loi le Chapelier : Bemerkungen zur Geschichte und möglichen Wiederentdeckung des Individuums », Kritische Justiz, 22 (2), p. 157-175.

Zabel, B. (2014) : «Das Recht der Institutionen », in : Seelmann, K. / Zabel, B. (éd.) : Autonomie und Normativität, Tübingen, Mohr Siebeck, p. 153-176.

\section{NOTES}

1. Cf. Elias (1976 [1939]).

2. Rousseau (1964 [1762]), p. 381.

3. Cf. Le Moniteur universel, III, 91 (cit. https://cnrtl.fr/etymologie/institution).

4. Le Moniteur Universel du 11 janvier 1790, p. 50.

5. La Chambre des Vacations remplissait les fonctions du Parlement régional dans les intervalles entre deux sessions de cet organe.

6. Le Moniteur Universel du 11 janvier 1790, p. 49.

7. Le Moniteur Universel du 13 janvier 1790, p. 57.

8. Sur l'individualisme de Le Chapelier, cf. Simitis (1989).

9. Montaigne (1962 [1850]), livre I, chap. xxvi, p. 154 sq. C'est pourquoi, jusque dans la langue du XVIII ${ }^{e}$ siècle, «institution" peut aussi avoir le sens d'« enseignement », et «instituer » celui d'« enseigner ». Sur cette signification du terme, cf. à nouveau Rousseau (1964 [1762]), livre II, chap. 7.

10. Zabel (2014). 
11. Au $\S 268$ des Principes, Hegel parle lui-même des «institutions qui subsistent au sein de l'État » dans le contexte de la rationalité « effectivement présente » (Hegel [2013], p. 438).

12. Cf. Hegel (1988), § 482, Remarque, p. 279 : « Si le savoir de l'Idée, c'est-à-dire du savoir qu'ont les hommes du fait que leur essence, leur but et leur objet est la liberté, si ce savoir est spéculatif, cette Idée elle-même est, en tant que telle, l'effectivité des hommes, non pas l'Idée qu'ils en ont, mais celle qu'ils sont. "

13. Cf. Dubiel (1976).

14. Cf. Henrich (1983), p. 30 sqq. et Honneth (2008), p. 106 sq.

15. Cf. Haferkamp (2018).

16. Au-delà de l'histoire terminologique, l'absence du concept d'institution pourrait aussi s'expliquer par le fait que Savigny, comme Hugo avant lui, est un interprète du droit romain, et reprend à ce titre la conception particulière des « institutions » qui a cours dans ce domaine (les Institutiones de Gaius, alors récemment découvertes), pour construire sur cette base une théorie scientifique autonome et une introduction méthodologique au travail de collection des sources, des lois et de la jurisprudence.

17. Fauconnet / Mauss (1901), p. 168 (rééd [1968], p. 17).

18. Hauriou (1933), p. 96.

19. Fauconnet / Mauss (rééd. 1968), p. 16.

20. Cf. Gehlen (1986), p. 101 sq.

21. Foucault (1966), p. 171 ; Foucault (1970), p. 167. Sur ce concept, cf. aussi Han (2003).

22. Pour reprendre le titre célèbre de l'ouvrage de Peter L. Berger et Thomas Luckmann (2018).

23. En « restant soigneusement sur le terrain des hypothèses ", Gehlen cherche à prévoir les effets du passage à « la culture industrielle » qui s'est engagé il y a deux cents ans : «[...] aucun secteur de la culture et aucun nerf du corps humain n'échappera à cette transformation qui peut encore durer des siècles, et dont il est impossible de prédire ce qui brûlera dans ce feu, ce qui se fondra en autre chose et ce qui résistera.» (Gehlen [1986], p. 263) Il évoque cependant le déclin de l'« art autonome » (ibid.), la montée d'une « opposition de grand style entre les races, peuples de couleur contre Blancs» (p.264), la diffusion d'un «luxe civilisationnel de masse dans l'humanité » entraînant vraisemblablement une régression du sens moral, qui marquera "peutêtre à son tour un progrès sur la voie d'une terrifiante naturalité » (p.135). Toutes ces perspectives sont dépeintes chez Gehlen sous le jour le plus sombre, même s'il lui arrive par ailleurs de prendre ses distances vis-à-vis d'auteurs "déclinistes » comme Oswald Spengler et Arnold Joseph Toynbee.

24. Gehlen (1986], p. 18.

25. Gehlen (1986), p. 61.

26. Gehlen (1986), p. 259.

27. Schelsky (1965), p. 33.

28. Schelsky (1965), p. 47.

29. Luhmann (2019), p. 13.

30. Luhmann (2019), p. 23.

31. Schelsky (1970), p. 25.

32. Cf. la description par Hegel du dernier stade de la Révolution, «le nœud [...] où achoppe l'histoire et qu'elle devra dénouer dans les temps futurs » : "Mécontent du fait que des droits rationnels, la liberté de la personne et de la propriété soient en vigueur, qu'il y ait une organisation de l'État et en celle-ci des sphères de la vie sociale qui ont à gérer eux-mêmes leurs affaires, que les gens sensés aient de l'influence sur le peuple et que chez celui-ci règne la confiance, le libéralisme oppose à tout cela le principe des atomes, des volontés particulières : tout doit se réaliser expressément par leur puissance et avec leur assentiment. Avec ce formalisme de la liberté, avec cette abstraction, on ne laisse rien de solide s'établir en fait d'organisation. » Hegel (1963), p. 343 (trad. modifiée). 
33. Schelsky (1970), p. 25.

34. Cf. à ce sujet ces phrases programmatiques de Castoriadis : "L'institution de la société est institution du faire social et du représenter/dire social. [...] La forme et l'orientation de cette institution peuvent, et doivent, changer; ce qu'elle crée-fabrique - l'individu social dans son mode d'être, ses références, ses comportements - aussi, sans quoi une révolution de la société est impossible ou condamnée à brève échéance à retomber dans "l'ancien fatras". " (Castoriadis [1975], p. 521 et 453).

35. Habermas (1985), p. 87.

36. Habermas (1985), p. 89.

37. Habermas (1985), p. 87.

38. Voir Göhler (1987) et (1997).

39. Cf. à ce sujet la théorie de la norme fondamentale chez Kelsen (1960), en particulier p. 196-226.

40. Sur la diversité de la pensée institutionnelle du droit, ses origines et sa possible actualité, cf. l'ensemble des contributions dans la Revue d'histoire des Facultés de droit, de la culture juridique, du monde des juristes et du livre juridique, $\mathrm{n}^{\circ} 32$ (2012).

41. Hauriou esquisse sa théorie de l'institution dans un article presque inconnu de 1906 : «L'institution et le droit statutaire », in : Recueil de législation de Toulouse 2 (1906), p. 134-182. Mais on trouve déjà de premières réflexions sur ce sujet dans "La science sociale traditionnelle " (1896) et «Leçons sur le mouvement social» (1898), deux textes repris dans Écrits sociologiques, Paris, Dalloz, 2008. Dans Principes de droit public, aussi (1 $1^{\text {ère }}$ éd. Paris, Sirey, 1910), le chapitre III est consacré à l'institution. L'idée de l'institution constitue donc le fil rouge de l'œuvre tout entier, bien que la thématisation «philosophique » complète n'en ait été donnée que dans l'article « La théorie de l'institution et de la fondation ... » de 1925.

42. Hauriou (1921), p. 14 et 82.

43. Hauriou (1933 [1925]), p. 101.

44. Hauriou (1933 [1928]), p. 76.

45. Hauriou (1933 [1925]), p. 101.

46. Hauriou (1933 [1925]), p. 108.

47. Hauriou (1933 [1925]), p. 101.

48. Hauriou (1933 [1925]), p. 120.

49. Vgl. Hauriou (1965 [1925]), p.38. La traduction allemande est quelque peu trompeuse, puisqu'en rendant «spéculation » tantôt par Handelsgeschäft (activité commerciale), tantôt par Gewinnerzielung ( réalisation de profit»), elle gomme la différence, visée par Hauriou, entre le but, la fonction et l'idée directrice. La phrase chez Hauriou est la suivante : « Les Anglais, quant à eux, ont complètement écarté la spécialité fonctionnelle pour leurs sociétés de commerce, toute société a vocation pour entreprendre n'importe quelle espèce de spéculation commerciale, parce que son idée directrice est la spéculation. » (Hauriou 1933 [1925], p. 100).

50. Il est pour le moins remarquable que la question de la morale des institutions, et donc aussi de la culpabilité et de la responsabilité des individus, ne joue aucun rôle dans les travaux de Gehlen et Schelsky, bien que le national-socialisme et les crimes commis par les Allemands sous ce régime, eussent largement fourni matière à une telle réflexion. 
INDEX

Schlüsselwörter : Institution, Macht, Gesellschaft, Moderne, Einleitung

Mots-clés : institution, pouvoir, société, modernité, introduction

\section{AUTEURS}

\section{JEAN-FRANÇOIS KERVÉGAN}

Jean-François Kervégan est professeur de philosophie émérite à l'Université Paris 1 PanthéonSorbonne. Pour plus d'informations, voir la notice suivante.

\section{CHRISTIAN SCHMIDT}

Christian Schmidt est Privatdozent à l'institut de philosophie de l'Université Humboldt à Berlin. Pour plus d'informations voir la notice suivante.

\section{BENNO ZABEL}

Benno Zabel est professeur de droit pénal et de philosophie du droit à l'Université de Bonn. Pour plus d'informations voir la notice suivante. 